\title{
A Planetary Perspective: The Commission on Comparative Planetology
}

\author{
by James W. Head, Alexander T. Basilevsky and Richard A.F. Grieve
}

The relatively small Commission on Comparative Planetology provides an example of another mode of operation for IUGS. CCP links other IUGS bodies on matters relating to planetary studies. It also stimulates research into the application of these studies to the investigation of the Earth itself. As a background to the Congress-wide colloquium on the 20th anniversary of the Apollo II landing, co-sponsored by $\mathrm{CCP}$ at the Washington IGC, the authors summarize some current ideas on planetary evolution and state their view of future trends in this most exciting new field. (Ed.)

\section{Introduction to the Commission}

The exploration of the planets and satellites in the last 25 years has changed these bodies from predominantly astronomical objects to geological objects. Missions by the United States, the Soviet Union, and more recently by the European Space Agency and Japan, and observations and analyses by scientists throughout the world have resulted in the beginning of an understanding of the processes that form and modify the surfaces and interiors of many of these objects. This has contributed to the development of some general themes in the geological evolution of planets and satellites. These themes have, in turn, added to our perception of geological activities in the first 50-75 of of Earth history, a period for which the evidence is largely missing on our planet.

The International Union of Geological Sciences, recognizing the potential of planetary studies for the geological sciences, established a Commission on Comparative Planetology (CCP) in 1984. The broad subject area is that of comparative planetology with emphasis, as appropriate, on the relevance of planetary studies to terrestrial geological problems and the use of planetary analysis techniques in the study of the Earth. The objectives of CCP are mainly achieved by sponsoring, promoting, assisting in the organization and participating in symposia workshops, research and publications at the national and international level.

The membership of CCP is relatively small, with twelve practicing planetary geoscientists. It is naturally biased towards the major participants in planetary exploration, with 50 \% of the members coming from the United States and the Soviet Union. Planetary studies are by their nature multidisciplinary, and the membership reflects this diversity in its expertise, though all have a common interest in planetary geological evolution. The members were also chosen to provide a liaison with national and international agencies, such as the National Aeronautic and Space Administration, Interkosmos and the European Space Agency, and scientific organizations such as the Meteoritical Society and COSPAR.

CCP co-sponsors several meetings a year. Among these is the annual Lunar and Planetary Science Conference, held at the NASA-Johnson Space Center in Houston, Texas. This is the premier annual meeting in planetary geoscience and attracts 700-800 scientists from approximately 20 countries. The Commission also organizes two symposia per year on the subject of comparative planetology. These are alternately held in the U.S. and Soviet Union and are attended by some $50-100$ scientists. These symposia provide a vehicle for exchange between U.S. and Soviet scientists and foster international cooperation in research. One such current project involves the exchange of samples from terrestrial impact structures for isotopic dating and determination of impactor type. This project is designed to provide data to test the recent hypothesis that the Earth is subjected to periodic cometary showers that regularly affect biospheric evolution through climatic change.

Internal Differentiation and Early History of the Planets

Absolute dating of lunar rocks and differentiated meteorites, combined with photogeological analysis of the surface of planets and satellites, has provided convincing evidence that the internal differentiation of planetary bodies was not a gradual process but occurred very early and very rapidly in planetary history. In fact, it was initated during the accretion of the bodies from pre-planetary matter. These observations also show that the early stages of planetary evolution were significantly affected by heavy "meteoritic" bombardment (Fig. 1). Still enigmatic is the problem of whether

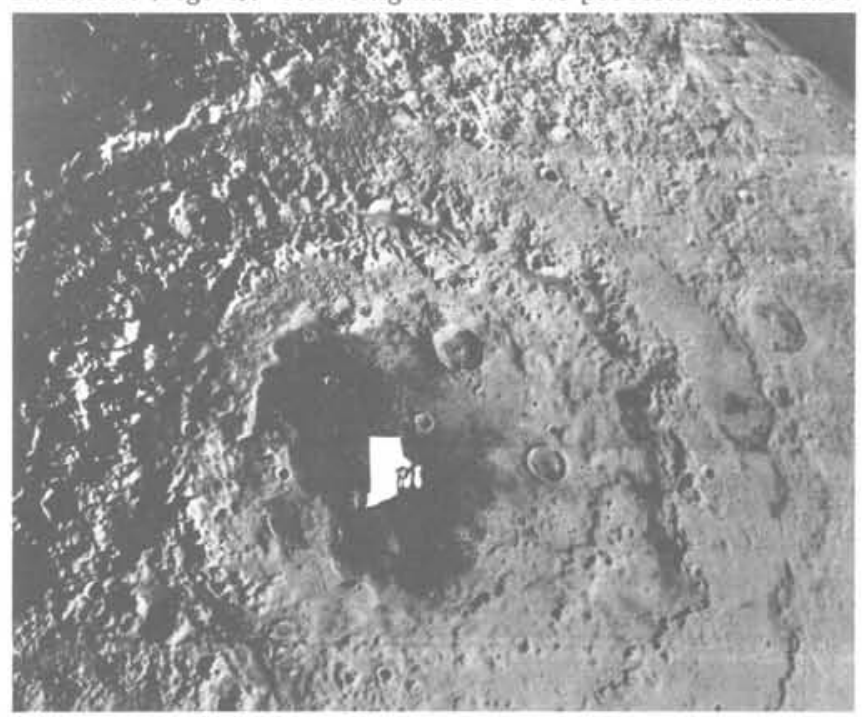

Figure 1: The Orientale impact basin on the Moon is about $900 \mathrm{~km}$ in diameter and formed about $4 \mathrm{Ga}$ ago. Such events modify the surface and interior of planets and represent zones of weakness that influence the geologic evolution of a planet for hundreds of millions to billions of years. The small white patch in the center of the basin is the outline of the state of Rhode Island, U.S.A. 
the well-documented bombardment episode, which occurred until $\backsim 3.9 \mathrm{Ga}$ ago on the Earth's Moon, was the gradual continuation of the planetary accretion process or was a newly generated cataclysm. In any event, impact cratering was an important process for the first few hundred million years of geological history.

The degree of preservation of this early phase of planetary history is also informative for the subsequent non-impact activity of individual bodies. For example, bodies that lost their internal heat relatively rapidly stabilized their crusts and lithospheres very early and became "one-plate" planets, preserving the record of heavy bombardment. The Moon, Mercury, and Mars are good examples of this. On the other hand, the larger Earth has segmented plates of crust and lithosphere being created at ridge crests, moving laterally, and being subducted. This produces and maintains a very young surface, with distinctive patterns of lateral deformation. As a result the record of the first half of Earth history, including the period of heavy bombardment, has been destroyed.

Comparative studies have led to the observation that larger bodies are more geologically active and evolved than smaller bodies. This correlation is evidently due to the heat generation/heat loss ratio, which is larger with the increasing body size. The impressive exception to this correlation is Jupiter's moon Io, where tidal interaction between it, massive Jupiter, and Europa, causes effective tidal heating. The extensive and currently active volcanism on Io, which is the size of the Earth's Moon, is, thus, driven by outside energy.

The continuing Voyager odyssey to distant parts of the Solar system has revealed a new geological world in the icy satellites of the outer planets. These also display the processes that have generally been considered as attributes of the internal character of the terrestrial planets: differentiation into compositionally different layers, volcanism and tectonism. The bright icy surfaces of some of these bodies, which have bulk densities typical of "dirty (silicate-rich)

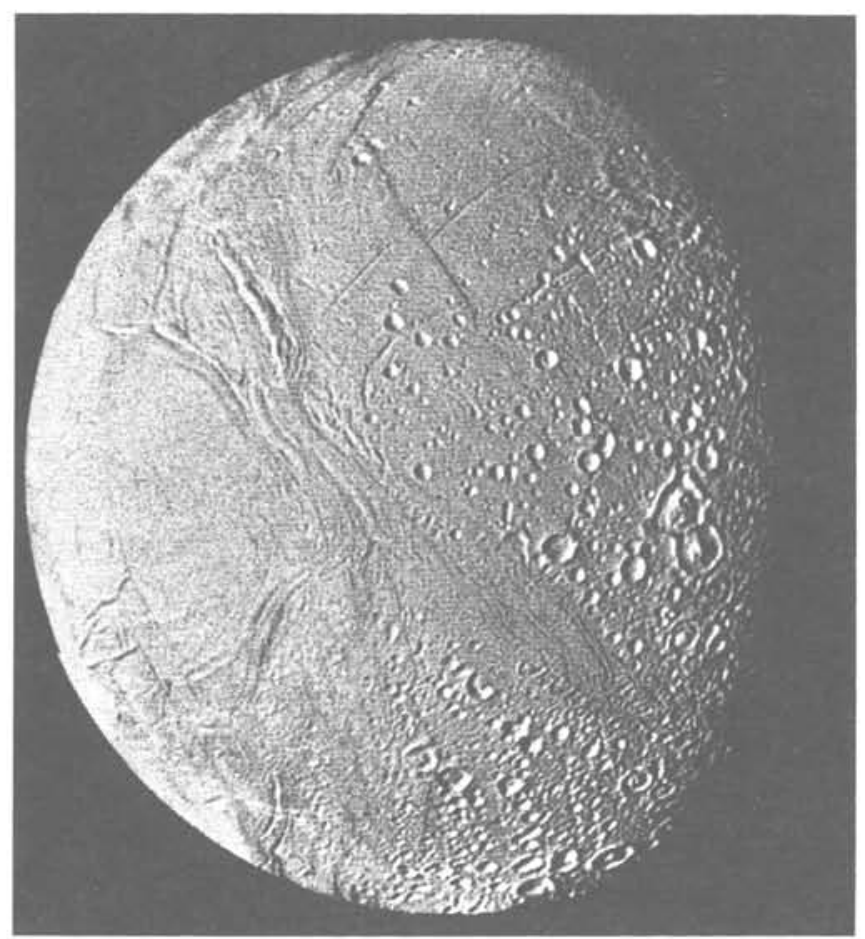

Figure 2: The surface of the Saturnian satellite Enceladus (about $500 \mathrm{~km}$ in diameter) has been modified by faulting and resurfaced by volcanism (smooth areas) in which the magma is composed mostly of liguid water. ice," imply internal differentiation into lighter ice and the denser silicate residual. Volcanism is represented by the eruption of liquid water (for example on Europa and Enceladus, Fig. 2), and tectonism is represented by the formation of extensional and/or compressional grooves and ridges (for example, Ganymede on Enceladus).

\section{Impacts, Voleanoes and Tectonics}

The relative contribution of impact cratering, volcanism and tectonism to geological evolution depends, in general, on the size of a body, but many aspects of their interrelations have yet to be studied. For example, it is clear that during the last $3.9 \mathrm{Ga}$, subsequent to the last of the major basinforming impacts, the role of impact triggering for tectonism and voleanism was negligible. But it may have been significant in the case of very large-scale impacts of earlier periods. The localization of mare basalts on the Earth's Moon inside the giant impact basins is very evident but as yet not well understood. These spatial relations are due to either hypsometric control, arising from the creation of topography by impact, or to impact-induced fracturing and heating. In the same way, it is difficult to ignore, yet difficult to prove, the relations of impact-induced fracturing with the later deformations of the mare basalts.

All of these observations have significant relevance for the Earth. The Earth must have shared the bombardment history of its planetary neighbours, and the influence of impact could have been no less significant on it. In fact, one of the leading hypotheses for the origin of our Moon is through the impact of a Mars-sized object on Earth very early in its history. This would have resulted in the neartotal melting of the Earth and the formation of the Moon from ejecta of Earth's mantle. As much as this hypothesis sounds like science fiction, it presently enjoys the support of the many geochemists, dynamicists, and geologists. Similarly, the potential role of impact in the biological evolution of the Earth has been brought to the attention of scientists by the hypothesis that extinctions at the CretaceousTertiary boundary may be the result of a major impact event.

Both volcanism and tectonism are the result of heating within a planetary body and are clearly related to each other. Among the small terrestrial planets, tha Moon has about $1 / 6$ of its surface covered with basaltic lavas and only a small number of tectonic features, predominantly located around the lava-flooded impact basins. These features appear to be related to the response of the lunar lithosphere to loading by lavas and subsequent flexure. This predominantly vertical style of tectonics is in contrast to the Earth. Mercury and especially Mars display both voleanic resurfacing and tectonic deformation of ancient and younger terrains. The Earth (with sediments ignored) and Venus, with their very abundant primarily volcanic and tectonic terrains, display the culmination of this sequence.

The innermost of the Gallilean satellites, Io, displays very active and almost global volcanic resurfacing with only small amounts of tectonic activity. Among the icy satellites the relationships between volcanism and tectonism are more complicated. In water-ice systems, the liquid is more dense than the pure solid, which makes volcanic phenomena dependent on the degree of impurity of ice, in terms of silicates, organic materials, etc. Most recently, the study of Halley's Comet confirmed that primitive cosmic ices have significant amounts of these components.

\section{Other Processes}

In addition to impact cratering, volcanism and tectonism, other processes may play a role in planetary geological evolution. These are driven mostly by solar energy and are significant on bodies with atmospheres. The presence of liquid water on or near the surface, as well as changes in the atmosphere and environment with time are important. 
Mars, with its abundant fossil, water-carved channels (Fig. 3) and evidence for a changing atmosphere with time, clearly offers a significant laboratory for the study of climate change. The role of large impacts in the evolution of the atmosphere, evidence for polar wandering, layering in the polar caps, the presence of water in the subsurface of Mars as revealed by crater ejecta patterns, and the sources and sinks for volatiles in the planet are all topics of current research. These issues are of extreme interest to our understanding of Earth, for although we know that the Earth's atmosphere has changed significantly with time, we have very little preserved record to study the causes of this change. This is one of the reasons that Mars is a significant target for future planetary exploration programs of the United States and the Soviet Union.

\section{Venusian Results}

In the last few years, most of the analysis of radar images and altimetry obtained by the Soviet Venera 15 and 16 space probes in 1983-84 has been done. The area surveyed covers the northern quarter of Venus (northward of $\backsim 30^{\circ} \mathrm{N}$ ). Radar image resolution is 1 to $2 \mathrm{~km}$, which provides the possibility of identifying landforms larger than several $\mathrm{km}$ across. Venera $15 / 16$ altimetry covers the north pole area, unsurveyed by previous missions, and provides an independent check for an area of overlapping Pioneer-Venus data. The results of the Venera survey are to be published in the form of radar mosaies, shaded relief, contour and geological maps at $1: 15,000,000$ scale by the U.S. Geological Survey. In addition, the results will be published in more detail, at the end of 1989 , in the form of a large album containing 27 quadrangle mosaics and geological maps reduced to $1: 10,000,000$ seale and some hundred sub-quadrangle mosaics at $1: 4,000,000$.

Photogeological analysis of the Venera radar data and altimetry indicates that basaltic volcanism and horizontal and vertical tectonic deformation are responsible for the majority of landforms and terrains observed. Some 150 craters ( 8 to $44 \mathrm{~km}$ in diameter) with typical impact crater morphology have been identified. At the global scale, their geological role was negligible, but their density permits one to estimate the average age of the terrains in the area surveyed. The estimated age is $0.5-1.0 \mathrm{Ga}$. This is significantly less than the average age of the terrains on the Moon but more than on Earth. This implies that the average resurfacing rate, due to volcanism and tectonics, on Venus is intermediate between the lunar and terrestrial ones. The morphological freshness of the observable landforms and consideration of the preservation state of the impact erater population implies a very low rate of externally caused resurfacing of Venus, compared to that of the Moon.

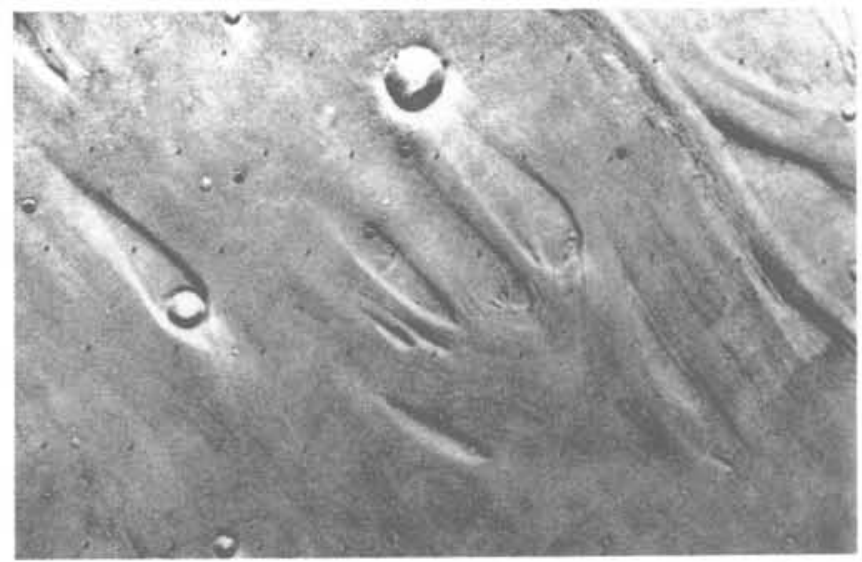

Fiqure 3: A Viking Orbiter view ( $35 \mathrm{~km}$ wide) of the surface of Mars showing evidence for the flow of liquid water on the surface in past martian

history. Liquid water is presently unstable on the surface of Mars.
Most of the basaltic volcanism in the area surveyed on Venus is represented by volcanic plains evidently formed by extensive fissure eruptions, but many volcanic edifices with characteristics typical of basaltic volcanism have also been observed. Among the tectonic features, large $(50-600 \mathrm{~km})$ ring-like structures (so-called arachnoids and coronae) have been observed. They have not been seen on any other planets, and their structural pattern resembles the ovoid tectonic features typical for the early Precambrian of Earth. These features and huge dome-like uplifts, with plains-like surface morphology, are evidently the result of hot-spot activity.

Very specific terrains, named tesserae (tile in Greek) and representing large areas of horizontal deformation, have been found mostly at high altitudes. Gravitational relaxation of uplands formed by other processes may be one of the main causes of the formation of these terrains. Some indirect evidence has been found that tesserae may be composed of non-basaltic (essentially feldspathic) material, being the compositional analog of terrestrial continents and lunar highlands. Belts of ridges with high relief (Fig. 4) and rises with low relief have also been seen, and are regarded as similar in formational processes to orogenic belts and mid-oceanic spreading centers on Earth.

\section{Thermal Processes}

The exploration of the planets has shown that the transfer of heat across the outer thermal boundary layer, the lithosphere, can take place in several ways. These include conduction (which has dominated heat transfer on the Moon, Mars, and Mercury for most of their history), advection (dominating the surface of lo with the transfer of heat directly to the surface through hot-spot volcanism), and crustal spreading and plate recycling (which dominates the heat transfer on Earth). Each of these mechanisms is characterized by distinctive styles of surface geology and volcanic and tectonic activiey. The smaller terrestrial planetary bodies are dominated by conduction, while the Earth is dominated by recycling of its lithospheric plates. Major questions remain as to the mechanisms of heat transfer that characterize the Earth-like (similar size, density, and position in the solar system) planet Venus and and the relevance this has for the earlier history of the Earth.

As outlined above, initial studies of Venus show evidence of large domal rises with rifts, associated volcanism, and large positive gravity anomalies (Beta Regio). Other areas show evidence for transform faults, topographic bilateral symmetry, and crustal spreading at annual rates of $1 \mathrm{~cm}$ or so (Western Aphrodite Terra). Still other areas show linear mountain belts that are very comparable to terrestrial orogenic belts (Western Ishtar Terra). Ishtar Terra may be the site of large-scale convergence and crustal imbrication and thickening.

Venus appears to be more similar to the Earth than to the smaller terrestrial planetary bodies. Because of its very high surface temperatures, it may have many similarities to the Earth's early Archean in terms of higher upper mantle temperature, thinner lithosphere, thicker "oceanic" crust at any spreading centers, lithospheric buoyancy and geometry, tectonic style, balance of forces at convergent zones, and process of crustal thickening and proto-continental growth. However, the global characterization of Venus in terms of volcanism and tectonism must await further data. The Magellan mission to Venus recently launched by the United States will provide high resolution (several hundred metres) radar images over almost the whole planet, as well as altimetry and gravity data. The Magellan mission has international investigators participating in the planning of the mission and analysis of the data, which will be available to the international scientific community. The results will be extremely important to our understanding of Venus, comparative planetology, and the history of the Earth. 


\section{Future Directions}

As we move into the next millennium, it is time to look into the future to examine trends in our discipline as a whole, and to see what our responsibilities are as planetary scientists and geologists in general. Four trends are clear. First, the Earth will be viewed more and more as a planet, as a global system of interacting processes. While this has been a common theme in global plate tectonics, the future will see this global view extended to the atmosphere, cryosphere, hydrosphere, and biosphere. Emphasis will be on the complex interaction of these domains (Earth Systems Science), and this will promote the development of a new paradigm for the interpretation of the terrestrial geological record. Planetary geologists, who naturally view the Earth as a planet, should be in the forefront of this trend, and can help to share this view with scientists in other disciplines.

Second, geologists will have at their disposal an almost overwhelming array of remote sensing techniques for studying the geology and other aspects of Earth systems. Because of the unfamiliar nature of many of these techniques to geologists studying the Earth, the incorporation of these techniques will be slow. Many planetary geologists, however, have used data from a wide range of the electromagnetic spectrum in mapping and solving geologic problems. We have the opportunity (and the responsibility) to be leaders in intergrating these techniques into terrestrial geologic studies.

Third, we will witness the resurgence of planetary exploration and the increased understanding of the Earth in the context of comparative planetology. The recent launch of the Magellan mission to Venus, and upcoming missions to Jupiter (Galileo), and Mars, (Mars Observer) will provide important data. The Soviet Union has defined an ambitious multi-mission exploration program and intensive study of Mars, culminating in the return of samples from Mars around the year 2000. The combination of the results of research from the last 25 years of exploration, and the more focused questions of the upcoming missions, will permit the

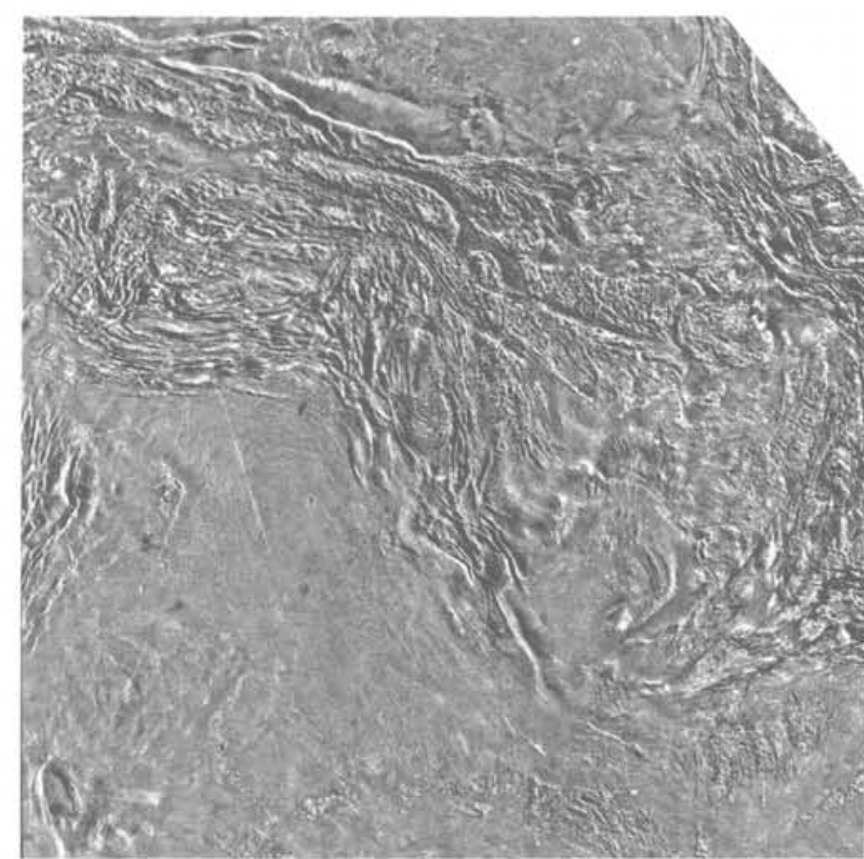

Figure 4: A radar image $(1500 \mathrm{~km}$ wide) of Akna Montes, an orogenic belt in northern Ishtar Terra, Venus, taken by the Venera 15/16 spacecraft. The anticlines and synclines are clear evidence of horizontal compressional deformation. A large volcano, Collette, with radiating flows is seen in lower left on Lakshmi Planum. development and testing of hypotheses for the origin and evolution of planets that will place the Earth in the context of its environment in the inner solar system. In addition, comparison of the Earth and its twin, Venus, will surely provide us with insight into the major processes of lithospheric evolution and, possibly, even the nature of the Earth in the first two-thirds of its history. We must work hard to make sure that the emerging lessons of comparative planetology are integrated into the geological sciences as a whole.

Finally, as the perspective of the Earth becomes more and more global, we find that we are dealing increasingly with the international geological community. From the point of view of planetary geology, the international aspects of solar system exploration have made interaction with international colleagues and other national agencies second-nature. We must do even more to promote this international view. In addition to supporting such efforts as the International Geological Congress, we must follow it with renewed dedication for specific interaction and sharing of data between countries.

The identification of these trends is clear. The challenge is to do the hard work necessary to forge the trends into reality, as we move into the next century of international cooperation in understanding the Earth and its neighborhood, the solar system.

Dr. J.W. Head is a Professor of Geological Sciences at Brown University (Providence, Rhode Island 02912 , U.S.A.). He is interested in volcanic and tectonic planetary processes and the relationships of planetary geology to the evolution of planetary interiors. He is the Chairman of CCP and is an investigator on the Magellan mission to Venus.

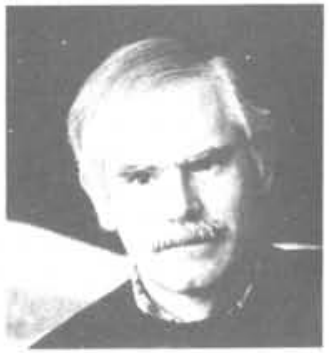

Dr. A.T. Basilevsky is the Chief of the Laboratory for Comparative Planetology of the Vernadsky Institute (Academy of Sciences, Kosyjin Street 19, 117975 GSP-1, Moseow V-334, U.S.S.R.). He is a specialist in cratering and in the geological evolution of planets and satellites. He is the Vice-chairman of CCP and an investigator on the Magellan mission to Venus.

Dr. R.A.F. Grieve is based in the Geophysics Division of the Geological Survey of Canada (1 Observatory Crescent, Ottawa, Canada K $1 A$ OY 3). The Secretary of CCP, he is interested in early crustal evolution and the use of data from terrestrial impact craters to constrain the geological effects and understand the physics of major impact events.
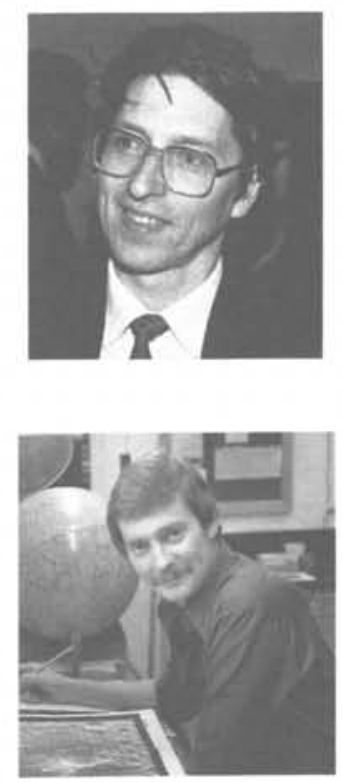